\title{
Regressions during reading: The cost depends on the cause
}

\author{
Michael A. Eskenazi ${ }^{1}$. Jocelyn R. Folk ${ }^{2}$
}

(C) Psychonomic Society, Inc. 2016

\begin{abstract}
The direction and duration of eye movements during reading is predominantly determined by cognitive and linguistic processing, but some low-level oculomotor effects also influence the duration and direction of eye movements. One such effect is inhibition of return (IOR), which results in an increased latency to return attention to a target that has been previously attended (Posner \& Cohen, Attention and Performance X: Control of Language Processes, 32, 531$556,1984)$. Although this is a low level effect, it has also been found in the complex task of reading (Henderson \& Luke, Psychonomic Bulletin \& Review, 19(6), 1101-1107, 2012; Rayner, Juhasz, Ashby, \& Clifton, Vision Research, 43(9), $1027-1034,2003)$. The purpose of the current study was to isolate the potentially different causes of regressive eye movements: to adjust for oculomotor error and to assist with comprehension difficulties. We found that readers demonstrated an IOR effect when regressions were caused by oculomotor error, but not when regressions were caused by comprehension difficulties. The results suggest that IOR is primarily associated with low-level oculomotor control of eye movements, and that regressive eye movements that are controlled by comprehension processes are not subject to IOR effects. The results have implications for understanding the relationship between oculomotor and cognitive control of eye movements and for models of eye movement control.
\end{abstract}

Jocelyn R. Folk

jfolk@kent.edu

1 Department of Psychology, Stetson University, DeLand, FL, USA

2 Department of Psychological Sciences, Kent State University, Kent, OH 44242, USA
Keywords Reading $\cdot$ Eye movements $\cdot$ Word recognition · Regressions

During reading, readers most often move their eyes forward in the text to process new information. These eye movements, or saccades, are necessary because visual acuity is limited to a small area, and only a certain amount of information can be extracted on each fixation (McConkie \& Rayner, 1976). However, not all eye movements take the eyes forward in the text. About $10 \%$ to $25 \%$ of eye movements move backwards in the text to reprocess information, which we will refer to as regressive eye movements or regressions (Rayner \& Pollatsek, 1989). These regressive eye movements are necessary to maintain comprehension as they allow readers to correct any misidentification or misinterpretation of the written text (Schotter, Tran, \& Rayner, 2014).

The fact that eye movements can move both forward and backwards depending on comprehension is one piece of evidence that direction of eye movements is mostly the result of cognitive and linguistic processing. For example, readers are more likely to make regressions to ambiguous homophones and heterophones, such as bank or tear, after reading disambiguating text (Duffy, Morris, \& Rayner, 1988; Folk \& Morris, 1995; Jones, Folk, \& Brusnighan, 2012). ${ }^{1}$ It is not only the direction of eye movements that is under cognitive control, but also the duration of eye movements. For example, readers spend more time processing words that are difficult because they are either unpredictable from the previous context or low frequency (Ehrlich \& Rayner, 1981; Rayner \& Duffy, 1986). Therefore, variations in both the direction and

\footnotetext{
${ }^{1}$ When readers do not make a regression to resolve ambiguity, data indicate that there is inflated reading time on the disambiguating region. However, for the current experiment, we are most interested in cases in which a regression is made to resolve the ambiguity.
} 
duration of eye movements can be explained by cognitive and linguistic processing.

While it is generally accepted that eye movements during reading are predominantly the result of cognitive and linguistic processing, low-level oculomotor factors also play an important role. One such oculomotor effect that is involved in reading is inhibition of return (IOR). IOR refers to the increased latency to return attention to a previously attended location than to a location that has not been recently attended (Posner \& Cohen, 1984). Most research involving IOR effects has involved nonreading visual processing tasks that rely on either reaction times or eye movements (for a review, see Klein, 2000). However, two studies have investigated whether IOR occurs during reading using eye movement measures (Henderson \& Luke, 2012; Rayner, Juhasz, Ashby, \& Clifton, 2003).

Rayner et al. (2003) investigated whether the low-level oculomotor effect of IOR is present in reading, a task that predominantly relies on higher-level cognitive processing. Results indicated that the IOR effect is not limited to simple tasks of visual search (Klein \& MacInnes, 1999), but is also involved in more complex tasks of word processing during reading. On the fixation prior to making a regression, readers took about $30 \mathrm{~ms}$ longer when the regression moved the eyes to a word that was previously fixated compared to a word that was previously skipped. Other research has also demonstrated the IOR effect in reading. Henderson and Luke (2012) investigated whether the IOR effect appears in both regular reading conditions and mindless reading of unreadable word blocks. The IOR effect appeared in both conditions, which the authors argued is evidence that the IOR effect in reading is the result of low-level oculomotor programming and not the result of comprehension and cognitive processes.

The purpose of the current experiments was to expand on these findings to determine whether IOR effects occur when regressions are the results of low-level oculomotor control, or from higher-level comprehension difficulties. Although the two previously mentioned experiments were well designed and provided clear evidence for IOR effects in reading, we take a different approach to investigating this issue. Our goal was to design two experiments that could disentangle regressions that were caused by comprehension issues and those that were caused by oculomotor error. The previous researchers attempted to eliminate saccades from global comprehension issues and those from oculomotor error by only looking at saccades that were launched between three and 15 characters from the target. However, we are attempting to isolate and investigate those issues separately in this work.

It is important to look at these two types of regressions separately because they are relying on two different processes. A regression that corrects for saccadic overshoot is relying on low-level oculomotor control, and a regression that is the result of comprehension difficulty is initiated as the result of cognitive processing. If IOR effects can be applied equally to all types of eye movements in reading, then they should be present in both types of regressions. Further, Klein and MacInnss (1999) suggested that IOR is a facilitative effect in that it prevents the reader from returning their eyes to a target that has already been processed. This explanation makes sense for some regressions, especially in visual search tasks, but when the regression is being initiated to resolve a comprehension problem in reading, it would not be facilitative to delay the execution of the regressive eye movement.

We created stimuli for two experiments to determine whether IOR effects are present when a regression is launched to correct for oculomotor error (Experiment 1) and when a regression is launched to correct for comprehension difficulty (Experiment 2). In Experiment 1, we adapted stimuli from Eskenazi and Folk (2015) because their target words resulted in a high (50\%) skipping rate and were often followed by an immediate corrective regression. In Experiment 2 we adapted stimuli from Titone (1998), which were lexically ambiguous homophones in which prior context was neutral but subsequent context supported the subordinate meaning by one disambiguating word. For example, in the sentence "Mike didn't like the grade of the hill that they were driving down," the word grade is lexically ambiguous and the word hill resolved the ambiguity to the subordinate meaning of slope. It is likely that readers will make a regression out of the word hill and into the word grade. This will allow us to determine whether IOR effects appear when regressions are made as a result of comprehension difficulties.

\section{Experiment 1}

\section{Method}

\section{Participants}

Fifty participants from Kent State University participated in this experiment for course credit. All participants had normal or corrected vision, spoke English as their first language, and had no reported reading disabilities.

\section{Stimuli}

Forty sentence stimuli were adapted from Eskenazi and Folk (2015) in that we used the original sentence frames, which were already normed for predictability. The sentence frames included a three-letter low-frequency unpredictable target word $\left(\right.$ word $\left._{n}\right)$ that was preceded by a five-letter high-frequency adjective ( $\left.\operatorname{word}_{\mathrm{n}-1}\right)$. We changed the sentences so that the 
word following the target word ( $\operatorname{word}_{\mathrm{n}+1}$ ) was always a fiveto seven-letter high-frequency preposition. Word frequencies were determined using CELEX database in the N-Watch program (Davis, 2005). An example sentence is below with an arrow indicating the path of the regression from $\operatorname{word}_{n+1}$ to $\operatorname{word}_{n}$.

\section{$\curvearrowleft$}

The actress wore a green gem around her neck at the award show.

These sentences were designed so that word $_{n}$ was likely to be skipped, which would increase the likelihood of an immediate regression from $\operatorname{word}_{n+1}$. The immediate regression would likely be a result of a corrective eye movement for overshooting the three-letter target word. This would allow us to compare reading times on $\operatorname{word}_{n+1}$ when the regression was to $\operatorname{word}_{n}$ based on whether $\operatorname{word}_{n}$ was skipped or fixated as a way to measure IOR.

We also included 10 filler sentences that each were followed by a comprehension question. This ensured that all readers were reading for comprehension. No participant scored below $80 \%$ accuracy, and the mean was $97 \%$ correct.

\section{Apparatus and procedure}

Eye movements were monitored using an EyeLink 1000 Plus eye tracker (SR Research). Eye movements were recorded from the right eye although viewing was binocular. Eye positions were measured using a sampling rate of $1000 \mathrm{~Hz}$. Participants were seated $60 \mathrm{~cm}$ (24 in.) from a computer monitor, and one degree of visual angle included four characters. Participants rested their chin and forehead on a head mount to reduce head movements. Participants used the left and right buttons of a keyboard to indicate that they have completed reading the sentence and to answer comprehension questions.

Prior to eye tracking, participants read and signed a consent form. Each participant's eye positions were calibrated and validated using a nine-point array on a 21-inch iMac computer screen. Prior to each trial, participants moved their eyes to a white circle on the computer screen, which performed a drift correction. Drift corrections were performed before each trial. After the drift correction was completed, the researcher controlled the onset of stimulus presentation by pressing a button on the eye-tracking computer. Participants were instructed to read at their own pace for comprehension. Stimuli were intermixed with filler sentences and comprehension questions randomly appeared on $25 \%$ of trials. Stimuli were presented in random order one at a time on a computer screen. At some points during the experiment the drift correct may have failed, in which case, participants were recalibrated if eye position error was greater than 0.3 degrees of visual angle.

\section{Results}

Fixations that were less than $80 \mathrm{~ms}$ and greater than $1,000 \mathrm{~ms}$ were eliminated from analyses. We also eliminated trials in which there was a blink or track loss in the areas of interest. This accounted for $2.3 \%$ of the data. Linear mixed effects (LME) analyses were conducted using the lme4 and language R packages in $\mathrm{R}$ Version 3.2.4 (Baayen, Davidson, \& Bates, 2008). All models included maximal random-effects structure (Barr, Levy, Scheepers, \& Tily, 2013). An alpha level of .05 was used, and significant effects were inferred when $t$ values were greater than 1.96 .

Only a subset of the data was analyzed because of the criteria used to define each condition. The two conditions were return regression (RR) and nonreturn regression (NRR). RR was defined as a regression that was made out of $\operatorname{word}_{n+1}$ and into $\operatorname{word}_{n}$ when $\operatorname{word}_{n}$ had previously been fixated. NRR was defined as a regression that was made out of $\operatorname{word}_{n+1}$ and into $\operatorname{word}_{n}$ when $\operatorname{word}_{n}$ had previously been skipped. Thus, RR was when the eyes returned to a previously attended location, and NRR was when the eyes returned to a location that was not previously attended. A regression was made out of $\operatorname{word}_{n+1}$ on $17 \%$ of all trials. On $89 \%$ of those trials the regression was made immediately into the target word $_{n}$, and on $10 \%$ of those trials it was made to an earlier part of the sentence. We only analyzed the $89 \%$ of trials in which the regression was made into the target word, so that we can be certain that the regression was made as a result of oculomotor correction. On those $89 \%$ of trials, RR accounted for $32 \%$ (85 trials) of the data and NRR accounted for $68 \%$ (180 trials) of the data. Analyses revealed that fixation durations on word $_{n+1}$ were significantly longer in the RR condition $(M=296 \mathrm{~ms}, S E=17 \mathrm{~ms})$ than in the NRR condition $(M=253 \mathrm{~ms}, S E=14 \mathrm{~ms}), \beta=41.69, S E=17.72, t=2.35$. Thus, the data are consistent with previous work in that readers take longer to return to a word that has been previously fixated, or IOR. ${ }^{2}$ In Experiment 2 we examined whether this effect also occurred when the cause of the regression was

\footnotetext{
${ }^{2}$ We also analyzed the frequency and length of word $_{n+1}$ to ensure that linguistic characteristics of this word did not contribute to these differences. Word ${ }_{n+1}$ in the RR and NRR condition had the same average length $\left(M_{\mathrm{RR}}=5.82, M_{\mathrm{NRR}}\right.$ $=5.94)$ and frequency $\left(M_{\mathrm{RR}}=475.93, M_{\mathrm{NRR}}=484.82\right)$.
} 
more likely a result of comprehension difficulties than from an immediate corrective regression to a previously fixated or skipped word.

\section{Experiment 2}

\section{Method}

\section{Participants}

Another sample of 50 Kent State University undergraduates participated in this experiment with the same set of controls as the first experiment.
Stimuli

The purpose of Experiment 2 was to determine whether IOR effects also appear when the regression is likely the result of reading comprehension difficulties rather than low-level oculomotor adjustment. Therefore, we adapted 45 sentence stimuli from Titone (1998) with lexically ambiguous target words. We used her sentence frames, but added in a disambiguating region that was most consistent with the subordinate interpretation of the ambiguous homophone. For example, the word grade is an ambiguous homophone in the sentence below, and the word hill is most consistent with grade's subordinate interpretation of slope:

Mike didn't like the grade of the hill that they were driving down.

Each sentence contained two short function words between the ambiguous homophone $\left(\operatorname{word}_{n}\right)$ and the disambiguating word $\left(\operatorname{word}_{n+1}{ }^{3}\right)$. Much like Experiment 1, this design allowed

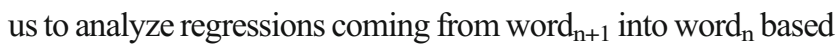
on whether $\operatorname{word}_{n}$ was fixated or skipped on the first pass through the sentence. However, the main difference now is that the regression is most likely caused by comprehension difficulty.

\section{Apparatus and procedure}

The apparatus and procedure were the same as Experiment 1. The only difference was that now there were 45 experiment stimuli and 15 filler sentences followed by comprehension questions. No participant scored below $80 \%$ accuracy, and the mean was $96 \%$ correct.

\section{Results}

The data were analyzed in the same way as Experiment 1. A return regression (RR) was defined as a regression out of word $_{n+}$ ${ }_{1}$, the disambiguating word, and into word $_{n}$, the ambiguous word, when it was previously fixated. A nonreturn regression (NRR) was defined as a regression out of word $_{n+1}$, the disambiguating word, and into $\operatorname{word}_{n}$, the ambiguous word, when it was previously skipped. A regression was made out of the disambiguating word $_{n+1}$ on $29 \%$ of all trials. On $83 \%$ of those trials the regression was made to the ambiguous target $\operatorname{word}_{n}$, and on $17 \%$ of those trials it was made to an earlier part of the sentence. We only analyzed the $83 \%$ of trials in which the regression was made into the ambiguous target word so that we can be certain that the

\footnotetext{
$\overline{3}$ Technically, word $_{n+1}$ is word $_{n+3}$, but we maintain this wording to avoid confusion between Experiments 1 and 2 . Word ${ }_{n+1}$ is always the word from which a regression was made.
}

regression was made as a result of comprehension difficulties. On those $83 \%$ of trials, RR accounted for $61 \%$ (1,112 trials) of the data and NRR accounted for $39 \%$ (711 trials) of the data. In contrast to Experiment 1, the analyses revealed no significant differences between the fixation duration on $\operatorname{word}_{\mathrm{n}+1}$ prior to the RR condition $(M=296 \mathrm{~ms}, S E=10 \mathrm{~ms})$ compared to NRR condition $(M=291 \mathrm{~ms}, S E=8 \mathrm{~ms}), \beta=7.18$, $S E=18.08, t=<1$. Thus, no IOR effect was observed when the regression was the direct result of comprehension difficulty. ${ }^{4}$

The presentation of a null effect is often problematic and should be met with some skepticism, as the conclusion could simply be a result of Type II error. To address this, we calculated the posterior probability in support of the null effect (Masson, 2011). This method relies on the Bayes factor and can quantify the degree to which a null effect is likely to be real, and not the result of Type II error. The results indicated that the probability of the null hypothesis given the data was $\mathrm{H} 0 \mid \mathrm{D}=.94$, which strongly supports the null effect. Thus, we can be confident that there is no inhibition to return the eyes to a previously processed word, when the regression was initiated as the result of comprehension difficulty.

\section{Discussion}

In a series of two experiments we attempted to determine whether IOR effects occur for the two main causes of regressive eye movements: (1) an immediate regression caused by oculomotor adjustment and (2) a regression caused by comprehension difficulty from misidentifying or misinterpreting a

\footnotetext{
${ }^{4}$ We also conducted analyses with the frequency and length of the disambiguating word as factors to ensure that linguistic characteristics of this word did not erase any possible effects. The inclusion of these factors did not change the results.
} 
previously read word. Previous work has indicated that lowlevel oculomotor IOR effects occur in the complex tasks of reading (Rayner et al., 2003) and mindless reading (Henderson \& Luke 2012), but we have taken a different approach by isolating the different causes of regressive eye movements. Therefore, we have expanded on previous research by demonstrating that IOR effects occur when a regressive saccade is initiated for oculomotor adjustment, but not when it is initiated to correct for a comprehension breakdown. ${ }^{5}$ These results have implications for understanding the connection between low-level and high-level control of eye movements during reading and for models of eye movement control.

Upon first discovering IOR effects in reading, Rayner et al. (2003) noted that it is striking that such a low-level oculomotor effect is present in such a complex task as reading. The duration and direction of eye movements in reading are determined primarily by linguistic processing, and it is therefore surprising that the duration of eye movements can also be determined by whether or not the eyes will be returning to a previously fixated word. However, they also noted that some other low-level effects have carried over into reading while others have not. For example, parallel programming of saccades, range effects, and center of gravity effects have all been observed in reading, while express saccades and the gap effect have not. The current results support the finding that low-level effects have carried over into reading, but suggest that higherlevel processing has dominance over these effects. When a regressive eye movement is initiated by the higher-level cognitive system rather than the lower level oculomotor system, the cost of making a regression is not present. This conclusion makes sense given that the goal of reading is to understand and comprehend the written text. One would not expect a mechanism to be included in this process that would delay or interfere with comprehension processes. The existence of IOR in other tasks such as visual search can be facilitative, as Klein and MacInnes (1999) suggest. It is beneficial to have some reluctance to move the eyes back to a target that has already been processed in visual search. In contrast, in reading it is necessary to sometimes move the eyes back to a target that has already been processed (Schotter et al., 2014).

Additionally, evidence from visual search experiments suggests that IOR is task-dependent (Dodd, Van Der Stigchel, \& Hollingsworth, 2009) and dependent on the relevance of the previously attended position (Farrell, Ludwig, Ellis, \& Gilchrist, 2010). Dodd et al., (2009) found that IOR only appeared in visual search tasks but found facilitation of return in other visual tasks such as free viewing and scene

\footnotetext{
${ }^{5}$ It is possible that IOR effects occur in other instances of regressions, or for other types of comprehension difficulty; however, the current data were designed to measure whether IOR effects occur for immediate corrective regressions and comprehension difficulty from misidentifying an ambiguous word.
}

memorization. They suggest that IOR can be turned off when it is not necessary to bias search away from novel targets. This is consistent with the current pattern of results, as it would not be beneficial to bias attention away from misidentified words. Farrell et al. (2010) provide a detailed explanation of how this process would work through an evidence accumulation model. When it is known, through repeated exposure, that a return saccade is necessary and beneficial, IOR is decreased. This could be applied to the current work in that readers may know that a regression is likely to be made to an ambiguous word because they are often misinterpreted (e.g., Folk \& Morris, 1995), therefore IOR would be decreased. This conclusion could be tested by investigating whether IOR effects occur on other commonly misidentified words.

These results also have implications for models of eye movement control. While earlier version of the E-Z Reader model focused almost exclusively on forward eye movements, recent advances to the model have begun to explain regressive eye movements (Reichle, Warren, \& McConnell, 2009). The current version of the model includes a $30 \mathrm{~ms}$ cost for all regressive saccades; however, the current data suggest a change to this cost. The data indicate that the cost could be higher than $30 \mathrm{~ms}$, or at least be flexible to include a range of costs. Clearly, the size of the IOR depends on the cause of the regression. However, we cannot definitively say that there is no cost associated with regressions initiated for comprehension difficulty, as we do not have a baseline comparison.

There are several points that could be argued against these new results. First, much of the conclusion rests on the null effect from Experiment 2, so we accept that these results should be viewed with some caution. However, the inclusion of our analysis of posterior probability of the null effect indicated that the lack of a difference between the two conditions is likely real. Second, one could argue that the lack of an IOR effect in Experiment 2 could be the result of expiration of an IOR because these effects are not permanent. Posner and Cohen (1984) found that IOR effects last up to two seconds, and therefore we would argue against this criticism because all of the regressive saccades were initiated in less than two seconds from leaving the word that would be the target of the regression. Third, there was a confound in that regressive saccades were significantly longer in Experiment $2(M=12.2)$ than in Experiment $1(M=4.8)$. However, previous work has found that saccade length has no correlation with fixation duration (Andriessen \& de Voogd, 1973), and thus the difference in saccade lengths is unlikely to be related to differences in reading times on $\operatorname{word}_{n+1}$.

Finally, one might question whether these two experiments are truly indexing different causes of regressions. However, there are several reasons to support that they are. First, the rate of return regressions in Experiment $2(61 \%)$ was almost twice as high as those in Experiment 1 (32\%). This suggests that 
readers were more likely to misidentify or misinterpret the target word and had to make a regression to that word to correct the misinterpretation. This conclusion is supported by the fact that readers are more likely to make regressions to ambiguous words that were misinterpreted than nonambiguous words (Jones et al., 2012). Second, readers make a corrective regression when the eyes overshoot, or move too far past the intended target, and this is more likely to occur when the eyes launch from a close target (McConkie, Kerr, Reddix, \& Zola, 1988). Experiment 1 was designed to induce this effect because readers launched their saccade from word $_{n-1}$ directly before the target word $_{n}$. Therefore, readers were moving their eyes back by one word to correct for the overshoot. In Experiment 2, readers were making a regression back three words, so this could not have been the result of oculomotor error, but rather comprehension difficulty.

In conclusion, the current experiment has provided further evidence that control of eye movements in reading is primarily guided by cognitive processing, but that low-level oculomotor control also plays a role in the duration of eye movements. While original reports of the IOR effect in reading appeared to suggest that this was predominantly a low-level effect, the current data suggest that higher-order cognitive processing can override this effect when the regressive eye movement is initiated to improve comprehension processes. The current results are limited to cases in which comprehension is disrupted from misidentifying a single ambiguous homophonic homograph, and therefore future work should investigate whether the IOR effect is present in other instances of comprehension difficulty including misidentifying heterophonic homographs or misidentifying entire phrases in garden path sentences.

\section{References}

Andriessen, J. J., \& De Voogd, A. H. (1973). Analysis of eye movement patterns in silent reading. IPO Annual Progress Report, 8, 29-34.

Baayen, R. H., Davidson, D. J., \& Bates, D. M. (2008). Mixed-effects modeling with crossed random effects for subjects and items. Journal of Memory and Language, 59(4), 390-412.

Barr, D. J., Levy, R., Scheepers, C., \& Tily, H. J. (2013). Random effects structure for confirmatory hypothesis testing: Keep it maximal. Journal of Memory and Language, 68(3), 255-278.

Davis, C. J. (2005). N-Watch: A program for deriving neighborhood size and other psycholinguistic statistics. Behavior Research Methods, $37(1), 65-70$.
Dodd, M. D., Van der Stigchel, S., \& Hollingworth, A. (2009). Novelty is not always the best policy inhibition of return and facilitation of return as a function of visual task. Psychological Science, 20(3), 333-339.

Duffy, S. A., Morris, R. K., \& Rayner, K. (1988). Lexical ambiguity and fixation times in reading. Journal of Memory and Language, 27(4), 429-446.

Ehrlich, S. F., \& Rayner, K. (1981). Contextual effects on word perception and eye movements during reading. Journal of Verbal Learning and Verbal Behavior, 20(6), 641-655.

Eskenazi, M. A., \& Folk, J. R. (2015). Skipped words and fixated words are processed differently during reading. Psychonomic Bulletin \& Review, 22(2), 537-542.

Farrell, S., Ludwig, C. J., Ellis, L. A., \& Gilchrist, I. D. (2010). Influence of environmental statistics on inhibition of saccadic return. Proceedings of the National Academy of Sciences, 107(2), 929-934.

Folk, J. R., \& Morris, R. K. (1995). Multiple lexical codes in reading: Evidence from eye movements, naming time, and oral reading. Journal of Experimental Psychology: Learning, Memory, and Cognition, 21(6), 1412.

Henderson, J. M., \& Luke, S. G. (2012). Oculomotor inhibition of return in normal and mindless reading. Psychonomic Bulletin \& Review, 19(6), 1101-1107.

Jones, A. C., Folk, J. R., \& Brusnighan, S. M. (2012). Resolving syntactic category ambiguity: An eye-movement analysis. Journal of Cognitive Psychology, 24(6), 672-688.

Klein, R. M. (2000). Inhibition of return. Trends in Cognitive Sciences, 4(4), 138-147.

Klein, R. M., \& MacInnes, W. J. (1999). Inhibition of return is a foraging facilitator in visual search. Psychological Science, 10(4), 346-352.

Masson, M. E. (2011). A tutorial on a practical Bayesian alternative to null-hypothesis significance testing. Behavior Research Methods, 43(3), 679-690.

McConkie, G. W., Kerr, P. W., Reddix, M. D., \& Zola, D. (1988). Eye movement control during reading: I. The location of initial eye fixations on words. Vision Research, 28(10), 1107-1118.

McConkie, G. W., \& Rayner, K. (1976). Asymmetry of the perceptual span in reading. Bulletin of the Psychonomic Society, 8(5), 365-368.

Posner, M. I., \& Cohen, Y. (1984). Components of visual orienting. Attention and Performance X: Control of Language Processes, 32, 531-556.

Rayner, K., \& Duffy, S. A. (1986). Lexical complexity and fixation times in reading: Effects of word frequency, verb complexity, and lexical ambiguity. Memory \& Cognition, 14(3), 191-201.

Rayner, K., Juhasz, B., Ashby, J., \& Clifton, C. (2003). Inhibition of saccade return in reading. Vision Research, 43(9), 1027-1034.

Rayner, K., \& Pollatsek, A. (1989). The psychology of reading. Englewood Cliffs: Prentice Hall.

Reichle, E. D., Warren, T., \& McConnell, K. (2009). Using EZ Reader to model the effects of higher level language processing on eye movements during reading. Psychonomic Bulletin \& Review, 16(1), 1-21.

Schotter, E. R., Tran, R., \& Rayner, K. (2014). Don't believe what you read (only once) comprehension is supported by regressions during reading. Psychological Science, 26(6), 1218-1226.

Titone, D. (1998). Hemispheric differences in context sensitivity during lexical ambiguity resolution. Brain and Language, 65(3), 361-394. 